\title{
The relationship between BSP mRNA expression and 25(OH)D/OPG in peripheral blood of newly diagnosed T2DM patients with different bone mass
}

\author{
Sitong Fan', Zhe Wang', Qi Li', Liya Luo'2, Yuyu Zhu², Yan Yang' \\ ${ }^{1}$ Department of Endocrinology, Affiliated Hospital of Zunyi Medical University, Guizhou Province, China \\ ${ }^{2}$ Department of Endocrinology, Zunyi Medical College Affiliated Hospital, Zunyi, China
}

\begin{abstract}
Introduction: The objective of the study was to detect the levels of osteoprotegerin (OPG) and 25-hydroxyvitamin D [25(OH)D], as well as the expression of bone sialoprotein (BSP) mRNA, in the peripheral blood of patients with newly diagnosed type 2 diabetes mellitus (T2DM) under different bone mass conditions, and to explore its role and significance in the development process of T2DM combined with osteoporosis (OP).

Material and methods: A total of 225 patients hospitalised in the Endocrinology Department and General Department from May 2017 to May 2018 were enrolled and categorised into five groups: the pure T2DM group (group A, 45 patients), the bone mass reduction group (group B, 45 patients), the T2DM + bone mass reduction group (group C, 45 patients), the OP group (group D, 45 patients), and the T2DM + OP group (group E, 45 patients); meanwhile, age-matched healthy subjects undergoing physical examination in our hospital were collected as the normal control group (group NC, 45 cases). Logistic regression analysis was used to analyse the influencing factors of bone mass in patients with T2DM.

Results: Compared with group B, the expression levels of glycated haemoglobin $\left(\mathrm{HbA}_{1 \mathrm{c}}\right), 25(\mathrm{OH}) \mathrm{D}, \mathrm{N}$-terminal propeptide of type I procollagen (PINP), fasting plasma glucose (FPG), fasting plasma insulin (FINS), high-density lipoprotein cholesterol (HDL-C), and BSP mRNA were significantly increased while OPG and b-collagen degradation products ( $\beta$-CTX) were significantly decreased in group A. Conclusions: The expression of BSP mRNA and the decrease of $25(\mathrm{OH}) \mathrm{D}$ and OPG in peripheral blood may participate in the development of diabetes and osteoporosis. (Endokrynol Pol 2020; 71 (2): 160-167)
\end{abstract}

Key words: type 2 diabetes mellitus; bone mass; bone sialoprotein; 25 hydroxy vitamin D; osteoprotegerin

\section{Introduction}

Diabetes mellitus (DM) is a group of metabolic diseases characterised by increased levels of chronic blood sugar caused by insulin secretion or defects [1]. Osteoporosis $(\mathrm{OP})$ is a systemic bone disease characterised by decreased bone mass, decreased bone strength, increased bone fragility, and proneness to fracture [2]. Bone sialoprotein (BSP) is a marker of bone metabolism that activates osteoblasts or osteoblast-like cells, guides bone mineralisation, and promotes bone formation [3]. Osteoprotegerin (OPG) is a glycoprotein secreted by osteoblasts, which plays an important role in maintaining normal bone metabolism [4]. Osteoprotegerin can significantly inhibit the differentiation and maturation of osteoclasts and bone resorption activity, and it might induce apoptosis and necrosis [5]. Vitamin D (VD) is closely related to the development of T2DM and OP. Its main function is to maintain normal blood calcium and phosphorus levels and promote normal bone min- eralisation [6]. 25-hydroxyvitamin $\mathrm{D}[25(\mathrm{OH}) \mathrm{D}]$ is stably present in the body and is a commonly used indicator for the observation of VD level. Studies have found that VD receptors are expressed on islet b cells; VD is involved in glucose metabolism, and VD deficiency impairs human glucose regulation and increases the risk of T2DM [7]. Under physiological conditions, VD is one of the essential substances for maintaining normal blood sugar and glucose-stimulated insulin secretion [8]. The relationship between BSP mRNA expression in peripheral blood and $25(\mathrm{OH}) \mathrm{D} / \mathrm{OPG}$ in type $2 \mathrm{DM}$ (T2DM) patients with different bone mass has not been reported in China or abroad. Therefore, this study intended to compare the expression of BSP mRNA in peripheral blood of healthy controls with that in T2DM patients with different bone masses, in order to explore the possible mechanisms of BSP mRNA, $25(\mathrm{OH}) \mathrm{D}$, and OPG in regulating glycometabolism, lipid metabolism, and bone mass, thus providing new ideas for early clinical prevention and treatment of DM and OP. 


\section{Material and methods}

\section{Subjects}

A total of 225 patients hospitalised in the Endocrinology Department and General Department from May 2017 to May 2018 were enrolled and categorised into five groups: the pure T2DM group (group A, 45 patients), the bone mass reduction group (group B, 45 patients), the T2DM + bone mass reduction group (group C, 45 patients), the OP group (group D, 45 patients), and the T2DM + OP group (group E, 45 patients); meanwhile, age/age-matched healthy subjects undergoing physical examination in our hospital were collected as the normal control group (group NC, 45 cases). The subjects in group NC all had normal bone density and glucose tolerance (by oral glucose tolerance test). The T2DM patients were in compliance with the diagnostic criteria of WHO Diabetes (1999). Informed consent was obtained from all subjects, and the study was approved by the Ethics Committee.

\section{Inclusion criteria}

There were four inclusion criteria:

1 - females with menopause over one year and males aged $\geq 60$ years, meeting the criteria for newly diagnosed T2DM;

2 - without serious organic diseases or acute/chronic complications of diabetes, with normal liver and kidney function;

3 - the subjects in group NC had no family history of diabetes and osteoporosis;

4 - without a history of smoking or alcohol abuse.

\section{Exclusion criteria}

\section{There were six exclusion criteria:}

1 - with previous severe heart, liver, kidney, or other organ or endocrine diseases;

2 - used drugs that may affect sugar, fat, or bone metabolism in the past month;

3 - with a clear history of infection in the past two weeks;

4 - suffering from rheumatoid arthritis, thyroid, parathyroid, adrenal gland, gonads, bone tumours, pituitary, or other diseases affecting bone metabolism; with fractures;

5 - used drugs that may affect bone metabolism (sex hormones, bisphosphonates, glucocorticoids, high-dose calcium, VD preparations, etc.) in the past six months;

6 - with a history of smoking or a long history of heavy drinking.

\section{Diagnostic criteria}

The diagnostic criteria for OP refer to the diagnostic criteria for the diagnosis and treatment of primary $\mathrm{OP}$ issued by the Osteoporosis and Bone Mineral Diseases Society of the Chinese Medical Association [9]. According to the DXA measurement results, the bone density being lower than and within the standard deviation of bone peak of healthy adults with the same sex and race is defined as normal; the reduction ranging from 1- to 2.5-fold standard deviation is defined as low bone mass; the reduction equalling or more than 2.5-fold standard deviation is defined as OP; the degree of bone density reduction meets the diagnostic criteria of OP, and severe OP is defined as OP simultaneously combing with one or more fragile fractures. Bone mineral density is usually expressed as $\mathrm{T}$-Score, $\mathrm{T}$-score $=($ measured value - peak bone density of normal young people with the same race and gender)/standard deviation of peak bone density of normal young people with the same race and gender. The diagnostic criterion of OP referring to the bone mineral density of central axis (lumbar vertebrae $1-4$, femoral neck or total hip) or $1 / 3$ of the distal radius based on DXA measurement is T-score $\leq-2.5$.

\section{Collection of general information}

The age and gender of all the study subjects were recorded, together with the height, weight, hip circumference, waist circumference,
Table 1. Specific primers used for real-time polymerase chain reaction (PCR)

\begin{tabular}{ll}
\hline Primer & Primer sequence (5'-3') \\
\hline \multirow{2}{*}{ BSP } & Upstream 5'-CAACGCCCTGACCACCGATAG-3' \\
& Downstream 5'-GGCTGCCTTCCGTCTCATAGT-3'
\end{tabular}

BSP — bone sialoprotein

and body mass index $\left(\mathrm{BMI}=\right.$ weight $[\mathrm{kg}] /$ height $\left.^{2}\left[\mathrm{~m}^{2}\right]\right)$. All the above data were imported into the database.

\section{Detection of general blood biochemistry and glucose metabolism indexes}

Each subject was fasted for 12 hours and water-inhibited for eight hours before tests, and then sampled fasting venous blood was left standing still for $1 \mathrm{~h}$, separated the serum by 10 -min centrifugation at $3000 \mathrm{r} / \mathrm{min}$, and stored at $-80^{\circ} \mathrm{C}$. Real Time PCR was used to detect the expression of BSP mRNA (Tab. 1). Fasting plasma insulin (FINS), b-collagen degradation products $(\beta$-CTX), type I-collagen amino end lengthening peptide (PINP), and $25(\mathrm{OH}) \mathrm{D}$ were determined by electrochemiluminescence (reagents were from Roche, German). One Olympus (AU2700) automatic analyser was used for detecting blood lipids; the hexokinase method (reagents were from Beckman Coulter Experimental System Co., Ltd.) was used for measuring fasting blood glucose (FPG); high-performance liquid chromatography (hPLC) was used to determine glycated haemoglobin $\left(\mathrm{HbA}_{1}\right.$ ) (reagents were from Bole, USA); homeostasis model of assessment for insulin resistance index [HOMA-IR = $=\mathrm{FPG} \times \mathrm{FINS} / 22.5]$.

\section{Determination of bone mineral density}

The orthotopic bone mineral density (BMD, expressed in $\mathrm{g} / \mathrm{cm}^{2}$ ) of the lumbar vertebrae (L1-4), as well as the BMD of the left and right proximal femur, including the femoral neck, greater tubercle, and Ward's triangle, were measured using a Medix90 DMS manufactured by MEDILINK, France.

\section{Statistical analysis}

Statistical analysis was performed using SPSS 19.0. The measurement data that conformed or were close to the normal distribution were expressed as mean \pm standard deviation $(x \pm s)$. The comparison among multiple groups was analysed by ANOVA. The baseline irregularity was treated by covariance. The comparison of two variables was performed by line correlation analysis, and Logistic regression analysis was used among multiple variables, with $p<0.05$ being considered as statistical significance.

\section{Results}

\section{Comparison of general data and clinical biochemical indexes}

1. The comparison of age, disease duration, gender, and BMI among groups showed no statistical significance $(\mathrm{p}>0.05)$.

2. Compared with group $B$, the expression levels of $\mathrm{HbA}_{1 c^{\prime}}$ 25(OH)D, PINP, FPG, FINS, and HDL-C were significantly increased while OPG and $\beta$-CTX were significantly decreased in group A $(p<0.05)$, but the difference in TC, TG, and HOMA-IR was not statistically significant $(\mathrm{p}>0.05)$. 
3. Compared with group $\mathrm{C}$, the expressions of PINP, $25(\mathrm{OH}) \mathrm{D}$, and HDL-C were significantly increased while OPG, $\beta$-CTX, FPG, and $\mathrm{HbA}_{1 \mathrm{c}}$ were significantly decreased in group $\mathrm{A}(\mathrm{p}<0.05)$, but the difference in TC, TG, FINS, and HOMA-IR was not statistically significant $(\mathrm{p}>0.05)$.

4. Compared with group $\mathrm{D}$, the expression levels of $\mathrm{HbA}_{1 c^{\prime}}$ PINP, 25(OH)D, FPG, HDL-C, and FINS were significantly increased while OPG and $\beta$-CTX were significantly decreased in group A $(p<0.05)$, but the difference in TC, TG, and HOMA-IR was not significantly different $(\mathrm{p}>0.05)$.

5. Compared with group $\mathrm{D}$, the expression levels of PINP, 25(OH)D, and HDL-C were significantly increased while OPG, $\beta$-CTX, FPG, and $\mathrm{HbA}_{1 \mathrm{c}}$ were significantly decreased in group $A(p<0.05)$, but the difference in TC, TG, FINS, and HOMA-IR was not statistically significant $(\mathrm{p}>0.05)$.

6. Compared with group $\mathrm{NC}$, the expression levels of OPG, FPG, $\mathrm{HbA}_{1 c^{\prime}}$ HOMA-IR, TG, TC, $\beta$-CTX, and FINS were significantly increased while the expressions of $25(\mathrm{OH}) \mathrm{D}, \mathrm{HDL}-\mathrm{C}$, and PINP were significantly decreased in group A $(\mathrm{p}<0.05)$.

7. Compared with group $\mathrm{C}$, the expression levels of PINP, 25(OH)D, and HDL-C were significantly increased while OPG, $\beta$-CTX, $\mathrm{HbA}_{1 \mathrm{c}^{\prime}}, \mathrm{FPG}$, and FINS were significantly decreased in group $B(p<0.05)$, but the difference in TC, TG, and HOMA-IR was not statistically significant ( $\mathrm{p}>0.05)$.

8. Compared with group $\mathrm{D}$, the expression levels of PINP, 25(OH)D, and HDL-C were significantly increased while OPG, $\beta$-CTX, and $\mathrm{HbA}_{1 \mathrm{c}}$ were significantly decreased in group $B(p<0.05)$, but the difference in TC, TG, FPG, FINS, and HOMA-IR was not statistically significant ( $\mathrm{p}>0.05$ ).

9. Compared with group $\mathrm{E}$, the expression levels of PINP, 25(OH)D, and HDL-C were significantly increased while OPG, $\beta$-CTX, $\mathrm{HbA}_{1 \mathrm{c}^{\prime}}$ FPG, and FINS were significantly decreased in group $B(p<0.05)$, but the difference in TC, TG, and HOMA-IR was not statistically significant $(\mathrm{p}<0.05)$.

10. Compared with group $\mathrm{NC}$, the expression levels of OPG, FPG, $\mathrm{HbA}_{1 \mathrm{c}^{\prime}}$ HOMA-IR, TG, TC, $\beta$-CTX, and FINS were significantly increased while the expressions of $25(\mathrm{OH}) \mathrm{D}, \mathrm{HDL}-\mathrm{C}$, and PINP were significantly decreased in group $B(p<0.05)$.

11. Compared with group $\mathrm{D}$, the expression levels of $\mathrm{HbA}_{1 c^{\prime}}$ PINP, 25(OH)D, FPG, HDL-C, and FINS were increased significantly while OPG and $\beta$-CTX were decreased significantly in group $C(p<0.05)$, but the difference in TC, TG, and HOMA-IR was not statistically significant $(\mathrm{p}>0.05)$.

12. Compared with group $\mathrm{E}$, the expression levels of PINP, 25(OH)D, and HDL-C were significantly in- creased while OPG, $\beta$-CTX, FPG, and $\mathrm{HbA}_{1 \mathrm{c}}$ were significantly decreased in group $C(p<0.05)$, but there was no significant difference in TC, TG, FINS, and HOMA-IR ( $\mathrm{p}>0.05)$.

13. Compared with group $\mathrm{NC}$, the expression levels of OPG, FPG, $\mathrm{HbA}_{1 c^{\prime}}$ HOMA-IR, TG, TC, $\beta$-CTX, and FINS were significantly increased while $25(\mathrm{OH}) \mathrm{D}$, HDL-C, and PINP were significantly decreased in group $C(p<0.05)$.

14. Compared with group $\mathrm{E}$, the expression levels of PINP, 25(OH)D, and HDL-C were significantly increased while OPG, $\beta$-CTX, $\mathrm{HbA}_{1 c^{\prime}}$ FPG, and FINS were significantly decreased in group $\mathrm{D}(\mathrm{p}<0.05)$, but the difference in TC, TG, and HOMA-IR was not statistically significant $(\mathrm{p}>0.05)$

15. Compared with group NC, the levels of OPG, FPG, $\mathrm{HbA}_{1 c^{\prime}}$ HOMA-IR, TG, TC, $\beta$-CTX, and FINS were significantly increased while $25(\mathrm{OH}) \mathrm{D}, \mathrm{HDL}-\mathrm{C}$, and PINP were significantly decreased in group $\mathrm{D}$ $(\mathrm{p}<0.05)$.

16. Compared with group $\mathrm{NC}$, the expression levels of OPG, FPG, $\mathrm{HbA}_{1 \mathrm{c}^{\prime}}$ HOMA-IR, TG, TC, $\beta$-CTX, and FINS were significantly increased while $25(\mathrm{OH}) \mathrm{D}$, HDL-C, and PINP were significantly decreased in group $E(p<0.05)$ (Tab. 2, Fig. 1).

\section{Level of BSP mRNA}

1. Compared with group B, BSP mRNA in the peripheral blood was significantly upregulated in group A ( $\mathrm{p}<0.05)$.

2. Compared with group C, BSP mRNA in the peripheral blood was significantly upregulated in group A $(p<0.05)$.

3. Compared with group D, BSP mRNA in the peripheral blood was significantly upregulated in group A ( $p<0.05)$.

4. Compared with group E, BSP mRNA in the peripheral blood was significantly upregulated in group A ( $\mathrm{p}<0.05)$.

5. Compared with group NC, BSP mRNA in the peripheral blood was significantly downregulated in group A ( $p<0.05)$.

6. Compared with group C, BSP mRNA in the peripheral blood was significantly upregulated in group $B$ $(\mathrm{p}<0.05)$.

7. Compared with group D, BSP mRNA in the peripheral blood was significantly upregulated in group $B$ $(\mathrm{p}<0.05)$.

8. Compared with group E, BSP mRNA in the peripheral blood was significantly upregulated in group $B$ $(\mathrm{p}<0.05)$.

9. Compared with group NC, BSP mRNA in the peripheral blood was significantly downregulated in group B ( $\mathrm{p}<0.05)$. 
Table 2. Comparison of general data and biochemical indexes among groups $(x \pm s)$

\begin{tabular}{|c|c|c|c|c|c|c|c|c|c|c|}
\hline Group & $\begin{array}{c}N \\
(\mathrm{M} / \mathrm{F})\end{array}$ & $\begin{array}{c}\text { Age } \\
\text { (years) }\end{array}$ & $\begin{array}{c}\text { BMI } \\
{\left[\mathbf{k g} / \mathbf{m}^{2}\right]}\end{array}$ & $\begin{array}{c}\mathrm{HbA}_{1 \mathrm{c}} \\
(\%)\end{array}$ & \multicolumn{2}{|c|}{$\begin{array}{c}\text { FPG } \\
{[\mathrm{mmol} / \mathrm{L}]}\end{array}$} & \multicolumn{2}{|c|}{$\begin{array}{c}\text { PINP } \\
{[\mathrm{ng} / \mathrm{mL}]}\end{array}$} & \multicolumn{2}{|r|}{$\begin{array}{c}\text { OPG } \\
{[\mathrm{ng} / \mathrm{L}]}\end{array}$} \\
\hline$N C(n=45)$ & $45(25 / 20)$ & $63.39 \pm 3.24$ & $22.33 \pm 2.15$ & $3.99 \pm 0.35$ & \multicolumn{2}{|c|}{$4.92 \pm 0.81$} & \multicolumn{2}{|c|}{$58.02 \pm 12.96$} & \multicolumn{2}{|c|}{$149.03 \pm 21.02$} \\
\hline$A(n=45)$ & $45(20 / 25)$ & $62.52 \pm 2.39$ & $24.31 \pm 2.31$ & $8.31 \pm 1.72^{1}$ & \multicolumn{2}{|c|}{$8.82 \pm 2.08^{1}$} & \multicolumn{2}{|c|}{$27.11 \pm 9.93^{1}$} & \multicolumn{2}{|c|}{$180.99 \pm 22.04^{1}$} \\
\hline$B(n=45)$ & $45(21 / 24)$ & $63.92 \pm 3.98$ & $23.47 \pm 2.14$ & $5.41 \pm 1.73^{1,2}$ & \multicolumn{2}{|c|}{$6.27 \pm 1.76^{1,2}$} & \multicolumn{2}{|c|}{$20.25 \pm 8.63^{1,2}$} & \multicolumn{2}{|c|}{$200.53 \pm 23.53^{1,2}$} \\
\hline$C(n=45)$ & $45(23 / 22)$ & $65.82 \pm 4.92$ & $23.93 \pm 2.71$ & $9.89 \pm 1.94^{1-3}$ & \multicolumn{2}{|c|}{$10.76 \pm 2.02^{1}$} & \multicolumn{2}{|c|}{$14.91 \pm 6.73^{1-3}$} & \multicolumn{2}{|c|}{$210.94 \pm 24.72^{1-3}$} \\
\hline$D(n=45)$ & $45(22 / 23)$ & $63.68 \pm 4.32$ & $23.65 \pm 2.47$ & $6.09 \pm 1.99^{1-4}$ & \multicolumn{2}{|c|}{$6.49 \pm 1.82^{1,2}$} & \multicolumn{2}{|c|}{$8.36 \pm 4.84^{1-4}$} & \multicolumn{2}{|c|}{$240.93 \pm 27.22^{1-4}$} \\
\hline$E(n=45)$ & $45(24 / 21)$ & $64.18 \pm 3.99$ & $24.34 \pm 2.83$ & $14.39 \pm 2.18^{1-5}$ & \multicolumn{2}{|c|}{$12.99 \pm 2.09^{1}$} & \multirow[b]{2}{*}{ MA-IR } & $3.75^{1-5}$ & \multicolumn{2}{|c|}{$260.94 \pm 25.99^{1-5}$} \\
\hline Group & $\begin{array}{c}\beta \text {-CTX } \\
{[\mathrm{ng} / \mathrm{mL}]}\end{array}$ & $\begin{array}{c}\text { TC } \\
{[\mathrm{mmol} / \mathrm{L}]}\end{array}$ & $\begin{array}{c}\text { TG } \\
{[\mathrm{mmol} / \mathrm{L}]}\end{array}$ & $\begin{array}{c}\text { LDL-C } \\
{[\mathrm{mmol} / \mathrm{L}]}\end{array}$ & $\begin{array}{c}\text { HDL-C } \\
{[\mathrm{mmol} / \mathrm{L}]}\end{array}$ & HOI & & \multicolumn{2}{|c|}{$\begin{array}{l}\text { 25(OH)D } \\
{[\mathrm{ng} / \mathrm{mL}]}\end{array}$} & $\begin{array}{c}\text { FINS } \\
{[\mathrm{ulU} / \mathrm{mL}]}\end{array}$ \\
\hline$N C(n=45)$ & $0.17 \pm 0.11$ & $4.81 \pm 0.38$ & $1.23 \pm 0.39$ & $2.23 \pm 0.42$ & $1.31 \pm 0.30$ & \multicolumn{2}{|c|}{$1.98 \pm 0.09$} & \multicolumn{2}{|c|}{$34.96 \pm 2.39$} & $9.09 \pm 1.81$ \\
\hline$A(n=45)$ & $0.52 \pm 0.09^{1}$ & $6.91 \pm 1.31^{1}$ & $3.11 \pm 1.29^{1}$ & $3.42 \pm 0.37^{1}$ & $0.69 \pm 0.23^{1}$ & \multicolumn{2}{|c|}{$5.59 \pm 0.26^{1}$} & \multicolumn{2}{|c|}{$26.43 \pm 5.87^{1}$} & $\begin{array}{c}13.01 \pm \\
2.35^{1}\end{array}$ \\
\hline$B(n=45)$ & $\begin{array}{l}0.68 \pm \\
0.19^{1,2}\end{array}$ & $7.53 \pm 1.02^{1}$ & $3.32 \pm 1.03^{1}$ & $3.53 \pm 0.63^{1}$ & $\begin{array}{l}0.66 \pm \\
0.26^{1,2}\end{array}$ & \multicolumn{2}{|c|}{$\begin{array}{l}3.86 \pm \\
0.24^{1,2}\end{array}$} & \multicolumn{2}{|c|}{$\begin{array}{c}20.64 \pm \\
5.04^{1,2}\end{array}$} & $\begin{array}{c}10.53 \pm \\
1.46^{1,2}\end{array}$ \\
\hline$C(n=45)$ & $\begin{array}{l}0.72 \pm \\
0.31^{1-3}\end{array}$ & $7.33 \pm 1.31^{1}$ & $3.21 \pm 1.32^{1}$ & $3.58 \pm 0.77^{1}$ & $\begin{array}{l}0.51 \pm \\
0.32^{1-3}\end{array}$ & \multicolumn{2}{|c|}{$5.62 \pm 0.33^{1}$} & \multicolumn{2}{|c|}{$\begin{array}{c}14.67 \pm \\
4.241,2,3\end{array}$} & $\begin{array}{c}12.76 \pm \\
1.89^{1}\end{array}$ \\
\hline$D(n=45)$ & $\begin{array}{l}0.84 \pm \\
0.33^{1-4}\end{array}$ & $6.99 \pm 1.32^{1}$ & $3.26 \pm 1.36^{1}$ & $3.38 \pm 0.47^{1}$ & $\begin{array}{l}0.36 \pm \\
0.31^{1-4}\end{array}$ & \multicolumn{2}{|c|}{$\begin{array}{l}3.37 \pm \\
0.22^{1,2}\end{array}$} & \multicolumn{2}{|c|}{$9.96 \pm 4.24^{1-4}$} & $\begin{array}{c}10.36 \pm \\
1.64^{1,2}\end{array}$ \\
\hline$E(n=45)$ & $\begin{array}{l}0.87 \pm \\
0.42^{1-5}\end{array}$ & $7.22 \pm 1.21^{1}$ & $3.12 \pm 1.33^{1}$ & $3.72 \pm 0.44^{1}$ & $\begin{array}{l}0.29 \pm \\
0.277^{1-5}\end{array}$ & \multicolumn{2}{|c|}{$5.05 \pm 0.19^{1}$} & \multicolumn{2}{|c|}{$7.18 \pm 3.84^{1-5}$} & $\begin{array}{c}12.48 \pm \\
3.03^{1}\end{array}$ \\
\hline
\end{tabular}

Groups: NC — normal control group; A — patients with pure T2DM; B — patients with bone mass reduction; $\mathrm{C}$ - patients with T2DM + bone mass reduction; $\mathrm{D}$ - patients with $\mathrm{OP} ; \mathrm{E}$ - patients with T2DM + OP; BMI — body mass index; $\mathrm{HbA}$ — haemoglobin; FPG — fasting plasma glucose; PINP — N-terminal propeptide of type I procollagen; OPG — osteoprotegerin; $\beta$-CTX $-\beta$-collagen degradation products; TC — total cholesterol; TG — triglycerides;

LDL-C - low-density lipoprotein cholesterol; HDL-C — high-density lipoprotein cholesterol; HOMA-IR — homeostasis model of assessment for insulin resistance index; 25(OH)D — 25-hydroxyvitamin D; FINS — fasting plasma insulin; T2DM — type 2 diabetes mellitus; OP — osteoporosis

Note: vs. group $N C ;{ }^{1} p<0.05$; vs. group $A,{ }^{2} p<0.05$; vs. group $B,{ }^{3} p<0.05$; vs. group $C,{ }^{4} p<0.05$; vs. group $D,{ }^{5} p<0.05$

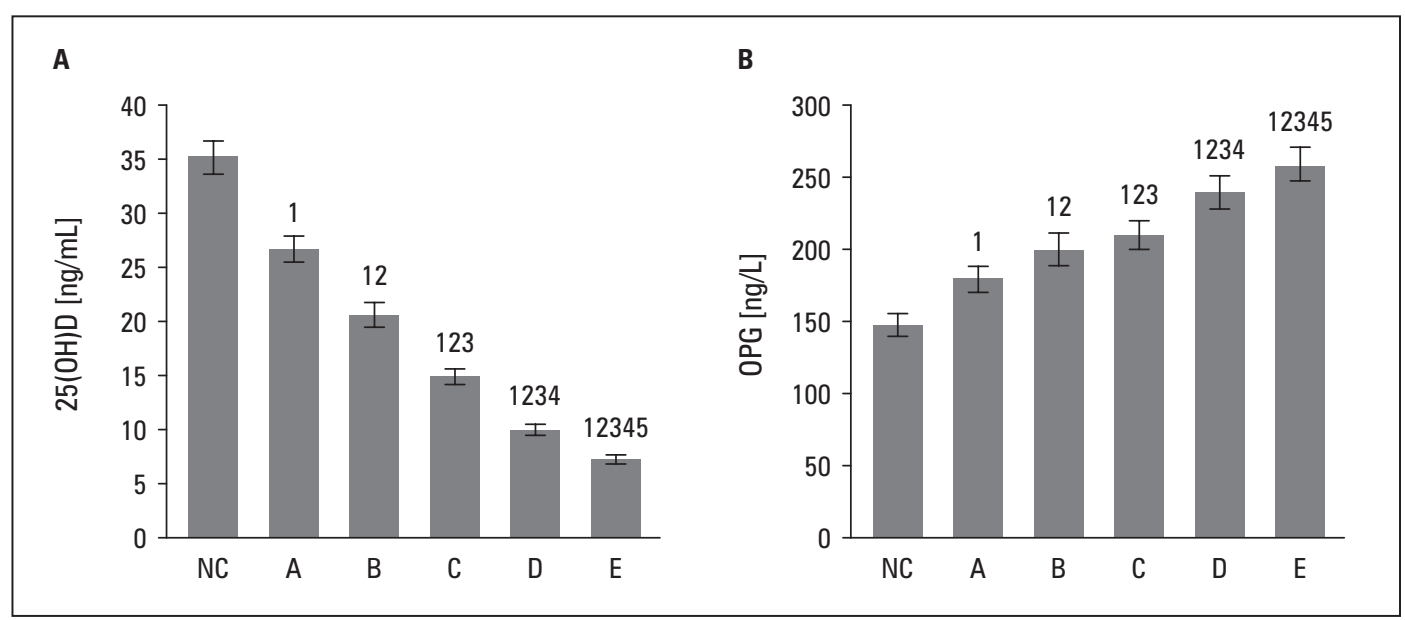

Figure 1A. Comparison of serum levels of 25-hydroxyvitamin D (25[OH]D) among groups; B. Comparison of serum osteoprotegerin $(O P G)$ levels among groups

10. Compared with group D, BSP mRNA in the peripheral blood was significantly upregulated in group $C$ $(\mathrm{p}<0.05)$.
11. Compared with group E, BSP mRNA in the peripheral blood was significantly upregulated in group $C$ $(\mathrm{p}<0.05)$. 
Table 3. Expressions of bone sialoprotein $(B S P) m R N A(x \pm s)$

\begin{tabular}{lcccccc}
\hline Group & NC & A & B & C & D & E \\
\hline BSP mRNA & $4.12 \pm 0.29$ & $4.02 \pm 0.35^{1}$ & $3.26 \pm 0.65^{1,2}$ & $2.71 \pm 0.75^{1-3}$ & $2.01 \pm 0.98^{1-4}$ & $1.07 \pm 1.11^{1-5}$ \\
\hline
\end{tabular}

Groups: NC — normal control group; A — patients with pure T2DM; B — patients with bone mass reduction; C — patients with T2DM + bone mass reduction; $\mathrm{D}$ - patients with $\mathrm{OP} ; \mathrm{E}$ - patients with $\mathrm{T} 2 \mathrm{DM}+\mathrm{OP}$

Note: vs. group NC, ${ }^{1} p<0.05$; vs. group $A,{ }^{2} p<0.05$; vs. group $B,{ }^{3} p<0.05$; vs. group $C,{ }^{4} p<0.05$; vs. group $D,{ }^{5} p<0.05$

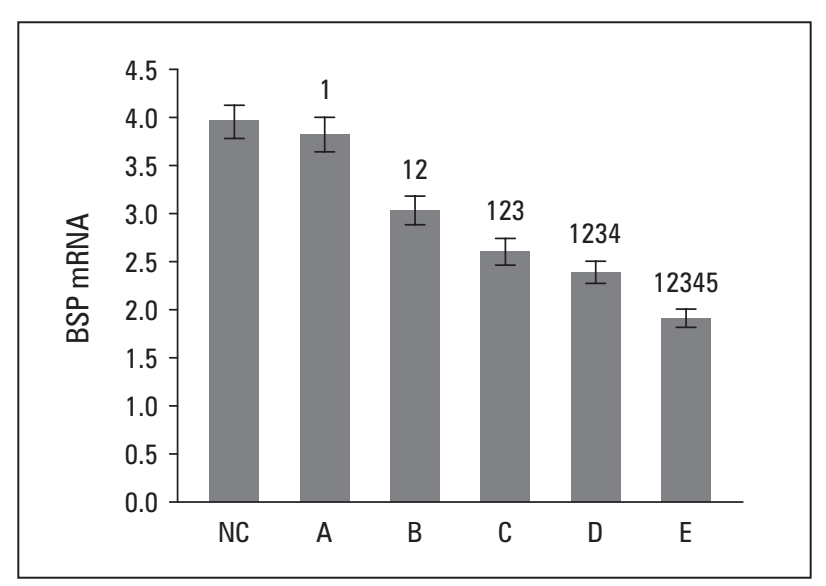

Figure 2 Comparison of expression levels of bone sialoprotein (BSP) $m R N A$ in peripheral blood among groups

12. Compared with group NC, BSP mRNA in the peripheral blood was significantly downregulated in group $\mathrm{C}(\mathrm{p}<0.05)$.

13. Compared with group E, BSP mRNA in the peripheral blood was significantly upregulated in group $D$ $(\mathrm{p}<0.05)$.

14. Compared with group NC, BSP mRNA in the peripheral blood was significantly downregulated in group D ( $\mathrm{p}<0.05)$.

15. Compared with group NC, BSP mRNA in the peripheral blood was significantly downregulated in group E ( $<$ < 0.05) (Tab. 3, Fig. 2).

\section{Comparison of $B M D$}

1. The comparison of BMD in the Ward's triangle and total hip was not statistically significant among groups ( $\mathrm{p}>0.05)$.

2. Compared with group B, BMD of L1-L4 and left femoral neck was significantly increased in group A ( $\mathrm{p}<0.05)$.

3. Compared with group C, BMD of L1-L4 and left femoral neck was significantly increased in group A ( $\mathrm{p}<0.05)$.

4. Compared with group D, BMD of L1-L4 and left femoral neck was significantly increased in group A $(p<0.05)$.
5. Compared with group E, BMD of L1-L4 and left femoral neck was significantly increased in group A ( $\mathrm{p}<0.05)$.

6. Compared with group NC, BMD of L1-L4 and left femoral neck was significantly decreased in group A ( $\mathrm{p}<0.05)$.

7. Compared with group C, BMD of L1-L4 and left femoral neck was significantly increased in group $B$ $(\mathrm{p}<0.05)$.

8. Compared with group D, BMD of L1-L4 and left femoral neck was significantly increased in group B $(\mathrm{p}<0.05)$.

9. Compared with group E, BMD of L1-L4 and left femoral neck was significantly increased in group $B$ $(\mathrm{p}<0.05)$.

10. Compared with group NC, BMD of L1-L4 and left femoral neck was significantly decreased in group B $(\mathrm{p}<0.05)$.

11. Compared with group D, BMD of L1-L4 and left femoral neck was significantly increased in group $C$ $(\mathrm{p}<0.05)$.

12. Compared with group E, BMD of L1-L4 and left femoral neck was significantly decreased in group $\mathrm{C}$ $(\mathrm{p}<0.05)$.

13. Compared with group E, BMD of L1-L4 and left femoral neck was significantly increased in group $D$ $(\mathrm{p}<0.05)$.

14. Compared with group NC, BMD of L1-L4 and left femoral neck was significantly decreased in group $D$ $(\mathrm{p}<0.05)$.

15. Compared with group NC, BMD of L1-L4 and left femoral neck was significantly decreased in group $\mathrm{E}$ $(\mathrm{p}<0.05)($ Tab. 4).

\section{Results of Pearson correlation analysis}

There was no statistical correlation in the level of BSP mRNA in peripheral blood with the age, FPG, BMI, TC, TG, LDL-C, FIns, or HOMA-IR ( $p>0.05$ ); the level of BSP mRNA in peripheral blood was negatively correlated with $\beta$-CTX, $\mathrm{HbA}_{1 \mathrm{l}^{\prime}}$ and OPG (correlation coefficients $r$ were $-0.483,-0.474$, and -0.527 , respectively, $\mathrm{p}<0.05$ ) (Tab. 2); the level of BSP mRNA in peripheral blood was positively correlated with $25(\mathrm{OH}) \mathrm{D}, \mathrm{PINP}$, 
Table 4. Comparison of bone mineral density (BMD) among groups

\begin{tabular}{lcccc}
\hline Group & L1-L4 $\left[\mathbf{g} / \mathbf{c m}^{2}\right]$ & Left femoral neck $\left[\mathbf{g} / \mathbf{c m}^{2}\right]$ & Ward's triangle $\left[\mathbf{g} / \mathbf{c m}^{2}\right]$ & Total hip $\left[\mathbf{g} / \mathbf{c m}^{2}\right]$ \\
\hline NC & $1.42 \pm 0.20$ & $1.27 \pm 0.17$ & $0.61 \pm 0.13$ & $0.78 \pm 0.12$ \\
\hline A & $1.33 \pm 0.18^{1}$ & $1.03 \pm 0.13^{1}$ & $0.59 \pm 0.11$ & $0.75 \pm 0.11$ \\
\hline B & $1.22 \pm 0.14^{1,2}$ & $0.97 \pm 0.09^{1,2}$ & $0.61 \pm 0.08$ & $0.80 \pm 0.09$ \\
\hline C & $1.19 \pm 0.11^{1-3}$ & $0.87 \pm 0.07^{1-3}$ & $0.60 \pm 0.09$ & $0.78 \pm 0.13$ \\
\hline D & $0.88 \pm 0.08^{1-4}$ & $0.68 \pm 0.06^{1-4}$ & $0.58 \pm 0.10$ & $0.81 \pm 0.08$ \\
\hline E & $0.69 \pm 0.06^{1-5}$ & $0.45 \pm 0.05^{1-5}$ & $0.62 \pm 0.12$ & $0.79 \pm 0.10$ \\
\hline
\end{tabular}

Groups: NC — normal control group; A — patients with pure T2DM; B — patients with bone mass reduction; C - patients with T2DM + bone mass reduction; $D$ - patients with $O P ; E$ - patients with $T 2 D M+O P$

Note: vs. group NC, ${ }^{1} p<0.05$; vs. group $A,{ }^{2} p<0.05$; vs. group $B,{ }^{3} p<0.05$; vs. group $C,{ }^{4} p<0.05$; vs. group $D,{ }^{5} p<0.05$

Table 5. Correlative analysis of bone sialoprotein (BSP) mRNA level in peripheral blood and clinical biochemical indexes ( $r$ )

\begin{tabular}{lcc}
\hline Item & BSP mRNA r & p \\
\hline $25(\mathrm{OH}) \mathrm{D}$ & 0.425 & 0.035 \\
\hline PINP & 0.477 & 0.041 \\
\hline $\mathrm{HDL}-\mathrm{C}$ & 0.626 & 0.036 \\
\hline OPG & -0.527 & 0.039 \\
\hline$\beta$-CTX & -0.483 & 0.027 \\
\hline $\mathrm{HbA}$ & -0.474 & 0.019 \\
\hline
\end{tabular}

25(OH)D - 25-hydroxyvitamin D; PINP — N-terminal propeptide of type I procollagen; HDL-C — high-density lipoprotein cholesterol; OPG — osteoprotegerin; $\beta$-CTX $-\beta$-collagen degradation products; $\mathrm{HbA}_{1 \mathrm{c}}-$ haemoglobin

and HDL-C (correlation coefficients $r$ were 0.425, 0.477, and 0.626, respectively, $\mathrm{p}<0.05$ ) (Tab. 5).

\section{Logistic regression analysis of risk factors for initial diagnosis of T2DM with OP}

Setting whether the newly diagnosed T2DM patient was associated with OP as the dependent variable, as well as the age, FPG, TC, TG, LDL-C, HDL-C, 25(OH)D, BSP mRNA, PINP, $\beta$-CTX, HbA ${ }_{1 c^{\prime}}$ HOMA-IR, and OPG as the independent variable, multivariate logistic regression analysis was performed targeting the groups. The results showed that $25(\mathrm{OH}) \mathrm{D}, \mathrm{PINP}$, and HDL-C were the independent protective factors for newly diagnosed T2DM patients with OP, but BSP mRNA, $\beta$-CTX, $\mathrm{HbA}_{\mathrm{lc}^{\prime}}$ and OPG were the independent risk factors for newly diagnosed T2DM patients with OP (Tab. 6).

\section{Discussion}

OP is a metabolic bone disease that causes bone fragility and proneness to fracture. Due to the increasing incidence in recent years, it has become a major disease that plagues the world [10]. T2DM is closely related to OP [11]. In recent years, studies have reported that BSP mRNA expression is closely related to DM and OP [12]. However, the expression of BSP mRNA in peripheral blood of T2DM patients with different bone mass has not been reported in China and abroad. Therefore, this study intended to compare the expression of BSP mRNA in peripheral blood of T2DM patients with different bone masses with that in healthy controls, aiming to explore the possible mechanisms of BSP mRNA, $25(\mathrm{OH}) \mathrm{D}$, and OPG in regulating glycometabolism, lipid metabolism, and bone mass, thus providing new

Table 6. Logistic regression analysis of risk factors associated with osteoporosis (OP) in patients with type 2 diabetes mellitus (T2DM)

\begin{tabular}{lcccc}
\hline Variable & Regression coefficient & Wald $\chi^{2}$ & $\mathbf{p}$ & OR (95\% CI) \\
\hline $\mathrm{HbA}_{1 \mathrm{c}}(\%)$ & 3.162 & 25.342 & 0.037 & $6.632(2.534-11.546)$ \\
\hline $\mathrm{BSP}$ mRNA & 7.352 & 3.353 & 0.025 & $7.135(2.174-9.234)$ \\
\hline$\beta-\mathrm{CTX}[\mathrm{ug} / \mathrm{L}]$ & 3.423 & 5.097 & 0.043 & $4.324(3.313-7.291)$ \\
\hline OPG [ng/L] & 5.211 & 3.119 & 0.041 & $3.391(1.192-5.273)$ \\
\hline $25(\mathrm{OH}) \mathrm{D}[\mathrm{ng} / \mathrm{mL}]$ & -0.931 & 5.128 & 0.017 & $0.425(0.219-0.816)$ \\
\hline PINP [ug/L] & -0.169 & 1.326 & 0.021 & $0.226(0.021-0.332)$ \\
\hline HDL-C [mmol/L] & -0.417 & 2.324 & 0.039 & $0.761(0.012-0.827)$ \\
\hline
\end{tabular}

$\mathrm{HbA}_{1 \mathrm{c}}$ - haemoglobin; $\mathrm{BSP}$ — bone sialoprotein; $\beta$-CTX — $\beta$-collagen degradation products; OPG — osteoprotegerin; $25(\mathrm{OH}) \mathrm{D}-25$-hydroxyvitamin $\mathrm{D}$; $\mathrm{PINP}$ - N-terminal propeptide of type I procollagen; HDL-C — high-density lipoprotein cholesterol 
ideas for early clinical prevention and treatment of DM and OP.

BSP is a kind of highly glycosylated, phosphorylated, and sulphated non-collagen protein containing a large amount of sialic acid and is mainly secreted and expressed by osteoblasts, osteoclasts, and cartilage tissue cells. BSP is specifically localised in bone tissues and plays a role in regulating the function of bone tissues [13]. Vitamin D plays an important role in bone metabolism. The main function of VD is to maintain normal blood calcium and phosphorus levels and to promote normal bone mineralisation [14]. Because $25(\mathrm{OH}) \mathrm{D}$ is relatively high in blood and has a longer half-life, the level of $25(\mathrm{OH}) \mathrm{D}$ is generally clinically determined so as to reflect the VD level in humans [15]. Osteoprotegerin plays a specific role in bone tissue and is a key determinant factor affecting the differentiation and maturation of osteoclasts, as well as regulating bone metabolism [16]. Osteoprotegerin inhibits the differentiation and maturation of osteoclasts, prevents excessive bone absorption, and plays a specific role in bone protection [17]. Studies have found that the serum OPG level in women aged 48-65 years is significantly high, indicating that the level in menopausal women is higher than that in non-menopausal women. It is speculated that the osteoclast function is active when oestrogen is deficient, resulting in bone resorption increase, bone loss acceleration, stimulated body compensatory bone formation increase, and corresponding increase in serum OPG [18]. b-collagen degradation products and PINP represent bone resorption and bone formation, respectively, and are commonly used biochemical indicators for reflecting bone transformation $[19,20]$. Factors such as hyperglycaemia, low HDL-C, hypertension, or endothelial injury can affect the expression of BSP mRNA [21]. This study also reveals that plasma 25(OH)D, PINP, and HDL-C are positively correlated with the expression of BSP mRNA in peripheral blood, but $\beta$-CTX, HbA ${ }_{\mathrm{lc}^{\prime}}$ HOMA-IR, and OPG are negatively correlated with BSP mRNA expression. With the decrease of bone mass, the levels of BSP mRNA, 25(OH) $\mathrm{D}, \mathrm{PINP}$, and HDL-C in serum decrease gradually (group $\mathrm{NC}>$ group $\mathrm{A}>$ group $\mathrm{B}>$ group $\mathrm{C}>$ group $\mathrm{D}>$ group $\mathrm{E}$ ), but the levels of $\mathrm{b}-\mathrm{CTX}$ and OPG gradually increase (group $\mathrm{NC}<$ group $\mathrm{A}<$ group $\mathrm{B}<$ group $\mathrm{C}<$ group $\mathrm{D}<$ group $\mathrm{E}$ ), which can presume that glucose- and lipid-metabolism disorder, as well as low levels of BSP mRNA and $25(\mathrm{OH}) \mathrm{D}$ in peripheral blood, can more easily induce OP. Therefore, decreased serum VD level, elevated OPG, and decreased expression of BSP mRNA in peripheral blood may be risk factors for predicting T2DM with OP.

\section{Conclusions}

Patients with T2DM and OP are often combined with lipid metabolism disorder, serum 25(OH)D level reduction, and gradual reduction of BSP mRNA expression in peripheral blood, resulting in loss of bone mass; vice versa, bone loss will further aggravate glucose- and lipid-metabolism disorder, which forms a vicious circle. Therefore, active VD supplementation may break this vicious circle. The decrease of $25(\mathrm{OH}) \mathrm{D}$ in serum may be involved in the development of DM and OP by down-regulating the expression of BSP mRNA in peripheral blood. Therefore, patients with T2DM should not only increase blood glucose control but also pay attention to serum $25(\mathrm{OH})$ $\mathrm{D}$ and BSP $\mathrm{mRNA}$ expression in peripheral blood, which may have a certain preventive effect on the prevention and treatment of DM and OP, but its specific mechanism needs further research and exploration.

\section{Acknowledgements}

This study was supported by the National Natural Science Foundation of China (Project No. 81460168); Subsidiary project for New Seedling Cultivation and Innovation Exploration of Zunyi Medical College (No. QKHRC[2017]5733-043); Joint Foundation of Zunyi (No. Zhengshi KHS [2018]63); Guizhou Provincial Natural Science Foundation (QKH-J [2020]1Y314).

\section{Conflicts of interest}

The authors declare no conflict of interest.

\section{References}

16. Riddy DM, Delerive P, Summers RJ, et al. G Protein-Coupled Receptors Targeting Insulin Resistance, Obesity, and Type 2 Diabetes Mellitus. Pharmacol Rev. 2018; 70(1): 39-67, doi: 10.1124/pr.117.014373, indexed in Pubmed: 29233848.

17. Khosla S, Hofbauer LC. Osteoporosis treatment: recent developments and ongoing challenges. Lancet Diabetes Endocrinol. 2017; 5(11): 898-907, doi: 10.1016/S2213-8587(17)30188-2, indexed in Pubmed: 28689769.

18. Zepp M, Kovacheva M, Altankhuyag M, et al. IDK1 is a rat monoclonal antibody against hypoglycosylated bone sialoprotein with application as biomarker and therapeutic agent in breast cancer skeletal metastasis. J Pathol Clin Res. 2018; 4(1): 55-68, doi: 10.1002/cjp2.88, indexed in Pubmed: 29416877

19. Daniele G, Winnier D, Mari A, et al. The potential role of the osteopontin-osteocalcin-osteoprotegerin triad in the pathogenesis of prediabetes in humans. Acta Diabetol. 2018; 55(2): 139-148, doi: 10.1007/s00592-017-1065-z, indexed in Pubmed: 29151224.

20. Rochette L, Meloux A, Rigal E, et al. The role of osteoprotegerin in the crosstalk between vessels and bone: Its potential utility as a marker of cardiometabolic diseases. Pharmacol Ther. 2018; 182: 115-132, doi: 10.1016/j. pharmthera.2017.08.015, indexed in Pubmed: 28867452.

21. Lips P, Eekhoff M, van Schoor N, et al. Vitamin D and type 2 diabetes. J Steroid Biochem Mol Biol. 2017; 173: 280-285, doi: 10.1016/j. jsbmb.2016.11.021, indexed in Pubmed: 27932304.

22. Norris JM, Lee HS, Frederiksen B, et al. TEDDY Study Group, TEDDY Study Group. Plasma 25-Hydroxyvitamin D Concentration and Risk of Islet Autoimmunity. Diabetes. 2018; 67(1): 146-154, doi: 10.2337/db17-0802, indexed in Pubmed: 29061729.

23. Wang H, Chen W, Li D, et al. Vitamin D and Chronic Diseases. Aging Dis. 2017; 8(3): 346-353, doi: 10.14336/AD.2016.1021, indexed in Pubmed: 28580189 
24. Chinese Medical Association Osteoporosis and Bone Mineral Disease Branch. Primary osteoporosis treatment guide. Chinese J. Osteoporosis Bone Min Dis. 2017; 10: 413-443.

25. Wright NC, Saag KG, Dawson-Hughes B, et al. The impact of the new National Bone Health Alliance (NBHA) diagnostic criteria on the prevalence of osteoporosis in the United States: supplementary presentation. Osteoporos Int. 2017; 28(11): 3283-3284, doi: 10.1007/s00198-017-4207-9, indexed in Pubmed: 28936598.

26. Hsu JY, Cheng CY, Hsu CY, et al. Type 2 diabetes mellitus severity correlates with risk of hip fracture in patients with osteoporosis. Neth J Med. 2018; 76(2): 65-71, indexed in Pubmed: 29515003.

27. DeShields SC, Cunningham TD, DeShields SC, et al. Comparison of osteoporosis in US adults with type 1 and type 2 diabetes mellitus. J Endocrinol Invest. 2018; 41(9): 1051-1060, doi: 10.1007/s40618-018-0828-x, indexed in Pubmed: 29353395.

28. Li C, Chen P, Duan X, et al. Bioavailable 25(OH)D but Not Total $25(\mathrm{OH})$ $D$ Is an Independent Determinant for Bone Mineral Density in Chinese Postmenopausal Women. EBioMedicine. 2017; 15: 184-192, doi: 10.1016/j. ebiom.2016.11.029, indexed in Pubmed: 27919752.

29. Wise SA, Phinney KW, Tai SSC, et al. Baseline Assessment of 25-Hydroxyvitamin D Assay Performance: A Vitamin D Standardization Program (VDSP) Interlaboratory Comparison Study. J AOAC Int. 2017; 100(5): 1244-1252, doi: 10.5740/jaoacint.17-0258, indexed in Pubmed: 28822355.

30. Mashavi M, Menaged M, Shargorodsky M, et al. Circulating osteoprotegerin in postmenopausal osteoporotic women: marker of impaired glucose regulation or impaired bone metabolism. Menopause. 2017 24(11): 1264-1268, doi: 10.1097/GME.0000000000000914, indexed in Pubmed: 28697041.

31. Chen C, Zheng H, Oi S, et al. Genistein and Silicon Synergistically Protects Against Ovariectomy-Induced Bone Loss Through Upregulating OPG/RANKL Ratio. Biol Trace Elem Res. 2019; 188(2): 441-450, doi: 10.1007/s12011-018-1433-8, indexed in Pubmed: 30014283.

32. Mamolini E, Cervellati C, Greco P, et al. VDR, RANKL and OPG polymorphisms as possible predisposing cofactors of postmenopausa osteoporosis: explorative study in Italian population. Gynecol Endocrinol. 2017; 33(12): 937-941, doi: 10.1080/09513590.2017.1323205, indexed in Pubmed: 28488893.

33. Yan J, Liu HJ Guo WC et al. Low serum concentrations of Irisin are associated with increased risk of hip fracture in Chinese older women. Joint Bone Spine. 2018; 85(3): 353-358, doi: 10.1016/j.jbspin.2017.03.011, indexed in Pubmed: 28408276.

34. Reginster JY, Sarkar S, Zegels B, et al. Reduction in PINP, a marker of bone metabolism, with raloxifene treatment and its relationship with vertebral fracture risk. Bone. 2004; 34(2): 344-351, doi: 10.1016/j.bone.2003.10.004 indexed in Pubmed: 14962813.

35. Yao W, Li X, Zhao B, et al. Combined effect of TNF- $\alpha$ and cyclic stretching on gene and protein expression associated with mineral metabolism in cementoblasts. Arch Oral Biol. 2017; 73: 88-93, doi: 10.1016/j.archoralbio.2016.09.013, indexed in Pubmed: 27710796. 